\title{
Orienting Attention to an Upcoming Tactile Event Involves a Spatially and Temporally Specific Modulation of Sensorimotor Alpha- and Beta-Band Oscillations
}

\author{
Freek van Ede, Floris de Lange, Ole Jensen, and Eric Maris \\ Donders Institute for Brain, Cognition, and Behaviour, Radboud University, 6500 HB Nijmegen, The Netherlands
}

\begin{abstract}
Our perception is facilitated if we know where and when a sensory stimulus will occur. This phenomenon is accounted for by spatial and temporal orienting of attention. Whereas spatial orienting of attention has repeatedly been shown to involve spatially specific modulations of ongoing oscillations within sensory cortex, it is not clear to what extent anticipatory modulations of ongoing oscillations are involved in temporal orienting of attention. To address this, we recorded magnetoencephalography while human participants performed a tactile discrimination task. We cued participants to the left or the right hand, after which a tactile stimulus was presented at one of several fixed temporal delays. We thus assessed whether and how ongoing sensorimotor oscillations are modulated during tactile anticipation. We provide evidence for three phenomena. First, orienting to an upcoming tactile event involves a spatially specific contralateral suppression of alpha- and beta-band oscillations within sensorimotor cortex. Second, this modulation is deployed with temporal specificity, and this is more pronounced for beta-band compared with alpha-band oscillations. Third, the contralateral suppression of beta-band oscillations is associated with faster responses to subsequently presented tactile stimuli. Control measures showed that these results cannot be explained by motor planning or execution. We conclude that the modulation of ongoing oscillations within sensory cortex reflects a unifying mechanism underlying both spatial and temporal orienting of attention.
\end{abstract}

\section{Introduction}

Understanding perception requires an understanding of the topdown influences that affect it. Anticipation is such a top-down influence, and it has been shown to involve a spatially specific modulation of ongoing oscillations within sensory cortex, before the presentation of a stimulus. For example, in anticipation of a lateralized visual stimulus, posterior alpha-band $(8-12 \mathrm{~Hz})$ oscillations are increased ipsilateral and decreased contralateral to the expected visual input (Worden et al., 2000; Sauseng et al., 2005; Kelly et al., 2006; Thut et al., 2006). We have recently observed a similar phenomenon in the tactile modality, i.e., expectation of a lateralized tactile stimulus involves an accordingly lateralized modulation of prestimulus sensorimotor beta-band (15-30 Hz) oscillations (van Ede et al., 2010).

Anticipatory modulation of ongoing alpha- and beta-band oscillations has consequences for neural processing and behavior. Neurophysiologically, these oscillations are inversely related to cortical excitability (Chen et al., 1998; Tamura et al., 2005; Romei et al., 2008; Sauseng et al., 2009). Behaviorally, they are inversely related to perceptual detectability (Ergenoglu et al., 2004; Hansl-

Received 0ct. 27, 2010; accepted Nov. 17, 2010.

We gratefully acknowledge the support of the BrainGain Smart Mix Programme of the Netherlands Ministry of Economic Affairs and the Netherlands Ministry of Education, Culture and Science. We thank Norbert Hermesdorf for designing and building the Braille tactile stimulation device and Pascal de Water for designing and building the electronics of this device.

Correspondence should be addressed to Eric Maris, Donders Centre for Cognition, Montessorilaan 3, 6525 HR Nijmegen, The Netherlands. E-mail: e.maris@donders.ru.nl.

DOI:10.1523/JNEUROSCI.5630-10.2011

Copyright $\odot 2011$ the authors $\quad 0270-6474 / 11 / 312016-09 \$ 15.00 / 0$ mayr et al., 2007; Romei et al., 2010), discriminability (van Dijk et al., 2008), and speed of visual and motor processing (Thut et al., 2006; Zhang et al., 2008; Pogosyan et al., 2009). These findings suggest a functional role of the anticipatory modulations: upcoming sensory processing will be enhanced when the sensory input arrives during a state of high excitability (involving contralaterally suppressed ongoing oscillations), and likewise intrusion of distracting input is blocked when arriving during a state of low excitability (involving ipsilaterally enhanced ongoing oscillations). Accordingly, the observed anticipatory modulations of ongoing oscillations within sensory cortex have been put forward as a physiological mechanism of spatial attention.

Perception is not only facilitated by knowledge of where a stimulus is likely to occur (spatial attention) but also by knowledge of when this stimulus is likely to occur (temporal attention) (Nobre, 2001). Like spatial attention, temporal attention is associated with modulations in brain activity (Nobre, 2001; Ghose and Maunsell, 2002; Nobre et al., 2007; Lakatos et al., 2008). However, it is at present unclear to what extent modulations in the amplitude of ongoing oscillatory activity are involved in temporal orienting of attention.

We hypothesized that a temporally specific modulation of ongoing oscillatory activity may constitute a physiological mechanism underlying temporal orienting of attention. Therefore, the modulation of ongoing oscillations, with specificity in both space and time, may be a unifying mechanism underlying both spatial and temporal selective attention. To address this, we recorded magnetoencephalography (MEG) while participants performed a tactile discrimination task. We cued participants to the left or 


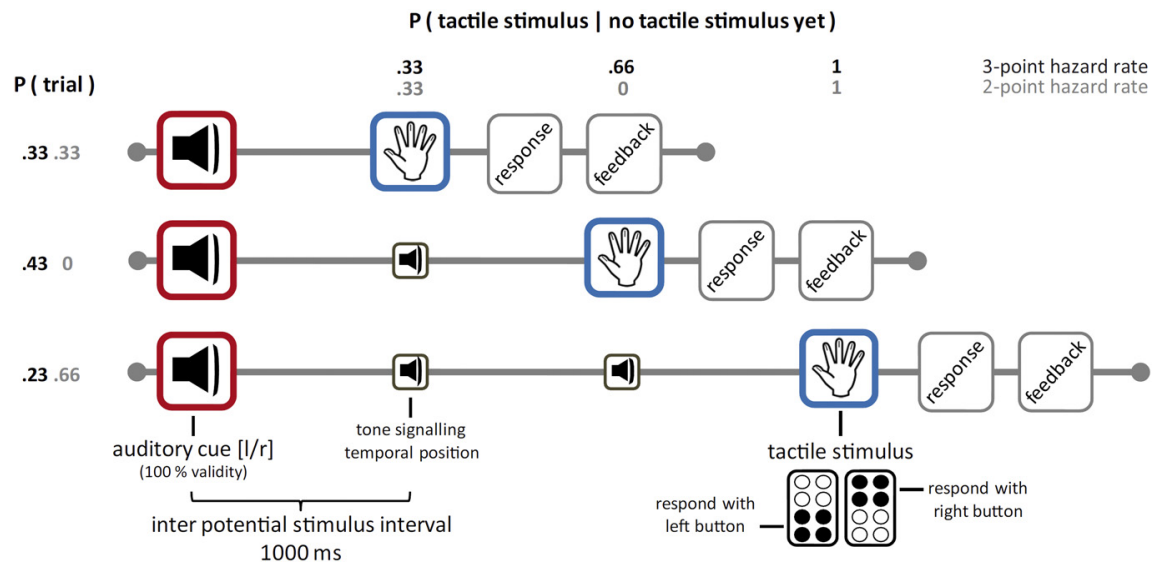

Figure 1. Experimental paradigm. Each trial starts with a randomly drawn auditory cue that indicates with $100 \%$ validity whether the tactile stimulus will be presented to the left or the right hand. In the three-point hazard rate condition, the tactile stimulation follows after 1, 2, or $3 \mathrm{~s}$, and in the two-point hazard rate condition, it follows after 1 or $3 \mathrm{~s}$. The probabilities as well as the hazard rates (conditional probabilities given that the stimulus has not been presented yet) can be read from the figure. When the tactile stimulus is not presented, a binaural auditory stimulus is presented that indicates the temporal position and thus signals that the tactile stimulus can occur 1 s later. When the tactile stimulus is presented, the upper four or the lower four pins of all Braille cells stimulating the fingertips of the cued hand are transiently raised. The participant is then required to discriminate between these alternatives (upper or lower pins) and respond by pressing the right or left button, respectively. Then, after $1 \mathrm{~s}$, feedback is presented. A correct response is followed by a single transient stimulation of both hands, whereas an incorrect response is followed by a double transient stimulation of both hands. At $1 \mathrm{~s}$ after the feedback, the next trial is presented.

the right hand, after which a tactile stimulus was presented at one of several fixed temporal delays. We thus investigated the spatial and temporal specificity with which ongoing sensorimotor oscillations are modulated during tactile anticipation. Additionally, we addressed whether such a modulation is constituted by a contralateral suppression or an ipsilateral enhancement. Finally, we assessed the behavioral consequences of prestimulus oscillatory activity.

\section{Materials and Methods}

\section{Participants}

A total of 20 healthy subjects voluntarily participated in the experiment (nine male; mean age, 26 years; range, 19-48 years). All participants provided written consent and were paid in accordance with guidelines of the local ethics committee (Committee on Research Involving Human Subjects, Region Arnhem-Nijmegen, The Netherlands).

\section{Experimental design and paradigm}

The experiment was designed to induce both spatial and temporal expectation. Figure 1 depicts the experimental paradigm. Every trial started with an auditory cue indicating whether the tactile stimulus would follow on the left or the right hand (with $100 \%$ validity). Left- and right-hand cues were randomly drawn for each trial. The cues for left- and righthand stimulation were a low $(500 \mathrm{~Hz})$ and high $(1000 \mathrm{~Hz})$ pitch pure tone. These tones had a duration of $50 \mathrm{~ms}$ and were binaurally presented via MEG-compatible air tubes. Their association with the side of tactile stimulation was counterbalanced across participants. After the cue, the tactile stimulus would be presented after 1,2 , or $3 \mathrm{~s}$ with probabilities governed by one of two hazard rate functions. The hazard rate is the conditional probability of observing an event (here, a tactile stimulus) given that it has not yet occurred. At the beginning of each trial, the subject does not know at which temporal position the tactile stimulus will be presented but, as the trial progresses, his expectation becomes more specific. Therefore, the behaviorally relevant quantity is the hazard rate, which is updated after every temporal position at which the stimulus does not occur. The first hazard rate function is characterized by the probabilities $0.33,0.43$, and 0.23 , which produce the hazard rates $0.33,0.66$, and 1 . The second hazard rate function is characterized by the probabilities $0.33,0.00$, and 0.66 , which produce the hazard rates $0.33,0.00$, and 1 (Fig. 1). In contrast with the first hazard rate, in the second hazard rate, the tactile stimulus is never presented at the second temporal position (i.e., $2 \mathrm{~s}$ after the cue). We used the above probabilities such that (1) the conditional probabilities would increase linearly with time and (2) both hazard rates would differ only at the second temporal position. The first and the second hazard rate functions are therefore called the three-point and the two-point hazard rate, respectively. It is crucial to note that a tactile stimulus is always presented at one of three possible temporal positions in a trial and never in between. In other words, we used discrete rather than continuous hazard rate functions. This allowed us to assess the temporal specificity with which anticipatory modulations of oscillations can be deployed. Because temporal specificity requires accurate mental timing, we assisted subjects by presenting a binaural $750 \mathrm{~Hz}$ pure tone at temporal positions at which the tactile stimulus did not occur.

Tactile stimuli were produced by piezoelectric Braille cells (Metec). A single Braille cell consists of eight pins, aligned in two series of four, that can be raised and lowered. Five such cells, together with a response button, were built into a graspable device (supplemental Fig. 1 , available at www.jneurosci.org as supplemental material), one for each hand. Tactile stimuli were produced by transiently raising the pins of all Braille cells overlying the five fingertips of a single hand. The pins were lowered into the cells again $20 \mathrm{~ms}$ after being raised, rendering the subjective experience of a tactile stimulus as a hit on the fingers.

When a tactile stimulus was presented, the participant was required to discriminate between two stimulus types, involving the stimulation of all digits of the hand with either the upper or lower four Braille cell pins. Participants responded by pressing the left button for the lower four pins and the right button for the upper four pins (Fig. 1). The two stimulus types (upper and lower pins) were equally often presented on the left and the right hand. As a result, our design was balanced with respect to the response side and the side of the tactile stimulus. After the response, feedback was presented. A correct response was followed after $1 \mathrm{~s}$ by a single tactile stimulus presented to both hands, whereas an incorrect response was followed by a double tactile stimulus (interstimulus interval of $200 \mathrm{~ms}$ ), also presented to both hands. The next trial was presented $1 \mathrm{~s}$ after the feedback.

The experiment consisted of two separate sessions that each contained 10 blocks. Each session lasted $\sim 1 \mathrm{~h}$. Within each block, 50 trials were presented. Within each session, six consecutive blocks contained trials in which the stimulus probabilities were governed by the three-point hazard rate function. The other four consecutive blocks contained trials in which the stimulus probabilities were governed by the two-point hazard rate function. The order of the two hazard rate suprablocks was counterbalanced across sessions and participants. Immediately before a change in hazard rate, participants were informed about this change. The complete experiment was programmed and run using the software package Presentation (Neurobehavioral Systems).

\section{$M E G$ recording}

The MEG system (CTF MEG TM Systems) contained 275 axial gradiometers and was housed in a magnetically shielded room. We also recorded bipolar surface EMG from both arms using two pairs of $\mathrm{Ag} / \mathrm{AgCl}$ electrodes. These electrode pairs were placed across the flexors of the forearm, with one electrode placed near the wrist and the other near the elbow, allowing us to measure digit a-specific contractions. Three localization coils, fixed to anatomical landmarks (nasion, left ear, right ear), were used to determine the position of the head relative to the gradiometers. All data were low-pass filtered by an anti-aliasing filter $(300 \mathrm{~Hz}$ cutoff), digitized at $1200 \mathrm{~Hz}$, and stored for offline analysis. No electro- 
oculography data were recorded because subjects were instructed to close their eyes during the experiment (in fact, during the experiment the lights were turned off).

\section{Data analysis}

Data were analyzed using FieldTrip (http://www.ru.nl/neuroimaging/ fieldtrip), an open-source Matlab toolbox developed at the Donders Institute for Brain, Cognition, and Behaviour (Nijmegen, The Netherlands).

All data epochs of interest were visually inspected, and those contaminated by artifacts were removed. Excessively noisy channels were also removed. Line noise was removed by means of the discrete Fourier transform (DFT). More specifically, we used the DFT to estimate the amplitudes and the phases of the 50,100, and $150 \mathrm{~Hz}$ components and subtracted the sine waves that were constructed from these DFTestimated amplitudes and phases. Finally, we removed the direct current component of the signals (which includes the offset of the superconducting quantum interference devices) by subtracting the mean of every epoch.

Channel and frequency band selection. For each participant, we selected a subset of channels as well as a frequency band on the basis of the stimulus-induced modulation in the poststimulus epoch. These selected channels were subsequently used for the analysis of the prestimulus epochs of interest. We now describe this channel and frequency band selection procedure, of which a graphical depiction is shown in supplemental Figure 2 (available at www.jneurosci.org as supplemental material). The procedure is based on the tactile stimulus-induced modulation of betaband oscillations that is known to originate from sensorimotor cortex (Salenius et al., 1997; Gaetz and Cheyne, 2006; van Ede et al., 2010). Specifically, we estimated beta power (see below, Frequency analysis) for all MEG channels in the range of $15-30 \mathrm{~Hz}$ and in the time window from 100 to $300 \mathrm{~ms}$ after stimulation, both of which were defined a priori. We then contrasted the beta power after left- and right-hand stimulation using the index $[($ right - left $) /($ right + left $)]$ and selected the 15 channels with the highest (lowest) index value, which were found over the right (left) sensorimotor cortex. This was done using the planar gradient (see below, Planar gradient calculation). Using these channels, we then calculated a single contra-over-ipsi power ratio (see below, Calculation of a contra-over-ipsi power ratio). To optimize the participant-specific selection of channels, we selected (on the basis of visual inspection) the participant-specific time window and frequency band of the poststimulus beta modulation and repeated the above steps. Importantly, the stimulus-induced beta modulation could be observed in all participants. On average, beta ranged between 13 and $31 \mathrm{~Hz}$ and was modulated between 120 and $400 \mathrm{~ms}$ after stimulus. Poststimulus modulations of alpha-band oscillations could not be observed in all subjects. For alpha, we therefore used a fixed frequency band of $8-12 \mathrm{~Hz}$.

Planar gradient calculation. Data were collected using an MEG system with axial gradiometers, which measure the first spatial derivative of the magnetic flux in the axial direction relative to the surface of the skull. From these axial gradiometer data, we calculated synthetic planar gradient data (Bastiaansen and Knösche, 2000). The planar gradient has the benefit that the activity is concentrated above the source (Hämäläinen et al., 1993). The synthetic planar gradient for a given channel consists of two components, of which one measures the spatial derivative along the anteroposterior axis of the MEG helmet and the other along the left-right axis. By linearly combining (rotating) these components (using coefficients that maximize the power of this linear combination), we obtained a single-component measure (in our case of oscillatory power) per channel. We refer to this as the planar gradient.

Calculation of a contra-over-ipsi power ratio. We based our calculation on a time-resolved power estimate (see below, Frequency analysis) for all selected channels. In the first step, the channels over the left and the right sensorimotor cortex were averaged separately. In the second step, for the left (right) sensorimotor channel, we calculated the ratio between the power during anticipation/stimulation of the right (left) hand and the power during anticipation/stimulation of the left (right) hand. In other words, we obtained a single left and a single right sensorimotor power ratio by dividing the oscillatory power during contralateral anticipation/ stimulation by the oscillatory power during ipsilateral anticipation/stimulation. In the third step, the left and the right power ratios were averaged, and the resulting measure will be denoted as the contra-overipsi power ratio. It is important to note that this measure is not affected by spatially a-specific effects of time, bilateral effects of potential motor preparation, and processing of the binaurally presented tones, because these effects are identical for channels ipsilateral and contralateral to the anticipated side.

Frequency analysis. We calculated oscillatory power estimates by means of the Fourier transform in combination with two tapering methods: a single Hanning taper and multiple discrete prolate spheroidal tapers (the multitaper method) (Percival and Walden, 1993). We calculated oscillatory power estimates both with and without time resolution. All calculations with time resolution involved a $300 \mathrm{~ms}$ sliding time window that was advanced in steps of $25 \mathrm{~ms}$. Power estimates were calculated for frequencies between 5 and $50 \mathrm{~Hz}$, and each one of them was based on a single Hanning taper. Non-time-resolved power estimates were calculated only for the frequency bands that were selected for the individual participants (see above, Channel and frequency band selection). For the participant-specific beta bands, power was estimated using the multitaper method, and for the alpha band, it was estimated using a single Hanning taper.

Source analysis. We calculated source-level power estimates by means of adaptive spatial filtering (beam forming). For every point source of interest, a source-level power estimate is calculated from a spatial filter that has unit gain for the point source of interest while maximally attenuating surrounding sources. This is accomplished by taking the crossspectral density matrix into account. This method is known as dynamic imaging of coherent sources (Gross et al., 2001; Liljeström et al., 2005), although we used it only for the estimation of source-level power. In practice, we set out by discretizing each individual's magnetic resonance image into a grid with $1 \mathrm{~cm}$ resolution. For each grid point, a lead-field matrix was calculated using a forward model based on a single shell volume conductor (Nolte, 2003). A spatial filter was then constructed for each point in the grid that was then applied to estimate the power at that source location. This power was subsequently contrasted between conditions of interest, analogous to the channel-level analyses. We were able to apply this beam-forming analysis to 17 of our 20 subjects. For the remaining three subjects, because of technical problems, no head localization was possible at the time of MEG data acquisition.

EMG analysis. Raw EMG traces were high-pass filtered ( $40 \mathrm{~Hz}$ cutoff) and subsequently rectified. We then calculated an ipsi-over-contra ratio, similar to the contra-over-ipsi ratio as was described for the MEG data. That is, we contrasted the high-pass-filtered and rectified EMG traces between two sets of trials: trials in which the (expected) event of interest occurs ipsilateral to the side of the EMG electrodes and trials in which it occurs contralateral.

Analysis of spatial specificity. We investigated whether orienting to an upcoming tactile event involves a spatially specific modulation of sensorimotor oscillations. We analyzed the first second after the cue for which we calculated the time-resolved contra-over-ipsi power ratio. We also constructed a topography of the difference between left- and right-hand expectation as described above for the poststimulus window. For this, we used a prestimulus window of $350 \mathrm{~ms}$ to stimulus onset. The choice for this window was based on a previous study (van Ede et al., 2010). Additionally, we analyzed the same (right - left) contrast in source space. Finally, for all channels, we linearly regressed time-resolved alpha- and beta-band power on time. This was done separately for anticipation of a left- and right-hand stimulus. The $t$ values of the resulting regression coefficients were taken as a normalized measure of the slope of alpha/beta power on time and averaged across participants.

Analysis of temporal specificity. We assessed whether the spatially specific modulation of sensorimotor oscillations is deployed with temporal specificity. We analyzed all trials for which the stimulus occurred at the last temporal position $(t=3)$. We calculated the time-resolved contraover-ipsi ratio with respect to power in the alpha and beta bands. This was done separately for both hazard rates. Temporal specificity was evaluated by means of two separate statistical analyses. First, we assessed whether the contra-over-ispi power ratio time courses differed between 
the three-point and the two-point hazard rate conditions. For this, we used a cluster-based permutation test. This test is well suited for controlling the false-alarm rate when facing multiple comparisons (as in our case, when conditions are contrasted for multiple time points). Specifically, the false-alarm rate is controlled by using a cluster statistic that is evaluated under a single permutation distribution (Maris and Oostenveld, 2007). Second, we assessed temporal specificity within each hazard rate. Temporal specificity should manifest itself as a stronger lateralization (indexed by our contra-over-ipsi power ratio) just before an expected event versus between two expected events. To test this hypothesis, we used the trials in which the stimulus was presented at $t=3$. We pooled the data points that estimated power in the last $300 \mathrm{~ms}$ before an expected event (three-point hazard rate: $t=0.85, t=1.85, t=2.85$; two-point hazard rate: $t=0.85, t=2.85)$ and contrasted this with a another measure that pooled the data points that estimated power between expected events (hazard rate $1: t=1.5, t=2.5$; hazard rate $2: t=2$ ). We evaluated this contrast by means of a paired sample $t$ test, treating the participants as the statistical replications.

Analysis of behavioral consequences. We investigated how the prestimulus oscillatory lateralization related to behavioral performance. For this, we separated for each participant both correct and incorrect trials as well as the fastest and the slowest trials (based on a median split) and analyzed these trials separately in the same ways as described above. We did this for the second that preceded the tactile stimulus to which the response was made. For statistical evaluation, we performed a cluster-based permutation test on the contra-over-ipsi power ratio time courses of the data that were separated according to behavioral performance.

\section{Results}

\section{Behavior}

Participants correctly discriminated, on average, $76 \pm 12 \%$ (mean $\pm \mathrm{SD}$ ) of the tactile stimuli and responded with an average latency of $1008 \pm 349 \mathrm{~ms}$. No differences in discrimination performance were observed for stimuli presented at the three different temporal positions $\left(F_{(2,18)}=1.3 ; p<0.286\right)$. In contrast, response times were significantly shorter for stimuli that were presented on a later compared with an earlier position $\left(F_{(2,18)}=\right.$ 9.1; $p<0.005$ ) (supplemental Fig. 3, available at www.jneurosci. org as supplemental material). Importantly, stimuli that are presented on a later position have a higher hazard rate (see Materials and Methods) than stimuli presented on an earlier position.

\section{Orienting to an upcoming tactile event involves a prestimulus suppression of contralateral sensorimotor alpha- and beta-band oscillations}

We assessed whether orienting to an upcoming tactile event involves a spatially specific modulation of sensorimotor oscillations. For this, we analyzed the first second after the cue that indicated whether the tactile stimulation would occur on the left or the right hand. Figure $2 \mathrm{~A}$ shows the time-resolved ratio of oscillatory power between contralateral and ipsilateral sensorimotor channels relative to the expected tactile stimulus. In anticipation of a lateralized tactile stimulus, there is less alpha-band $(8-12 \mathrm{~Hz})$ and beta-band $(15-30 \mathrm{~Hz})$ activity over the contralateral compared with the ipsilateral sensorimotor cortex. This effect is most pronounced for the beta band, and we will focus on this frequency band in the following. The corresponding results for the alpha band are highly similar and are shown in supplemental Figure 4 (available at www.jneurosci.org as supplemental material). This spatially specific modulation of prestimulus betaband oscillations is highly consistent across subjects, as is evident from Figure $2 B$ that shows the beta modulation together with its 95\% confidence interval. Additionally, the modulation becomes stronger toward the expected event, which is consistent with a role in sensory preparation.
Next, we investigated the spatial topography of the observed beta modulation. For every channel, we calculated the beta power in the last $350 \mathrm{~ms}$ preceding the time point of the first potential stimulus, separately for left- and right-hand anticipation, and subtracted the two. The resulting topography is shown in Figure $2 C$, together with the corresponding source reconstruction (see Materials and Methods). Over left sensorimotor cortex, beta power is lower in anticipation of a right-hand compared with a left-hand stimulus, whereas the reverse is true for the right sensorimotor cortex. The minimum and the maximum of the corresponding source-reconstructed power differences are both in the postcentral gyrus [i.e., primary somatosensory cortex (S1)].

These observations clearly show a spatially specific modulation of sensorimotor beta-band oscillations in anticipation of a tactile event. However, they do not distinguish between two possible constituents of such a modulation: a contralateral decrease or an ipsilateral increase in beta power. If the decrease over time in the contra-over-ipsi ratio of oscillatory power (depicted in Fig. $2 B$ ) is produced by a contralateral decrease, then the beta power over contralateral channels must decrease with time. Analogously, if it is produced by an ipsilateral increase, then the beta power over ipsilateral channels must increase with time. To investigate this, for all channels, we linearly regressed time-resolved beta power on time, separately for anticipation of a left- and right-hand stimulus. Figure $2 D$ depicts the results obtained from this analysis. In anticipation of a right-hand stimulus, the beta power over left sensorimotor channels decreases with time. Likewise, in anticipation of a left-hand stimulus, the beta power over right sensorimotor channels also decreases with time. This shows that the observed spatially specific modulation of sensorimotor beta-band oscillations is produced by a contralateral suppression.

It is important to note that this prestimulus beta modulation is highly similar to the observed poststimulus modulation (supplemental Fig. 5, available at www.jneurosci.org as supplemental material), in both terms of frequency content and spatial topography. This is suggestive of a common neural generator of the beta oscillation that is suppressed not only by tactile input but also by its anticipation. Moreover, the strength of the prestimulus beta modulation is almost as strong as the strength of the poststimulus modulation (compare Fig. $2 B$ with supplemental Fig. $5 B$, available at www.jneurosci.org as supplemental material). Note that these observations cast serious limitations to the use of prestimulus baseline corrections to predictable events (for the severe effect of prestimulus baseline-correcting-induced activity that follows an anticipated tactile event, see supplemental Fig. $5 D, E$, available at www.jneurosci.org as supplemental material). This correspondence between prestimulus and poststimulus oscillatory activity is specific to beta. In fact, in the poststimulus period, there is a lateralized modulation of gamma-band $(60-80 \mathrm{~Hz})$ oscillations (in accordance with Bauer et al., 2006) that was not observed in anticipation of a tactile stimulus (supplemental Fig. $5 D$, available at www.jneurosci.org as supplemental material).

It is important to rule out that the anticipatory beta modulation is related to motor planning or behavior. With respect to motor planning, it is important to note that the expected side of the tactile stimulus and the expected side of the button-press response were balanced. That is, a left (right)-hand stimulus required as often a left hand as a right-hand button press. Furthermore, no bias was observed with respect to the side of the button press in relation to the side of the stimulation (side congruent side incongruent responses: $t_{(19)}=-1.02 ; p=0.32$ ). To control for expectation-related motor behavior (such as contracting the hand on which the tactile stimulus was expected to occur), we 
recorded EMG activity over both arms. We observed no significant difference between ipsilateral and contralateral EMG activity during tactile anticipation. This null finding is unlikely to be attributable to an insensitive EMG measure because lateralized button presses as well as explicitly requested contractions resulted in significant differences between ipsilateral and contralateral EMG activity (supplemental Fig. 6, available at www. jneurosci.org as supplemental material). On the basis of these considerations, we argue that the observed beta modulation is related to anticipation of an upcoming tactile event. Accordingly, we argue that this modulation occurs at least partially within primary somatosensory cortex.

\section{Anticipatory modulations of ongoing sensorimotor oscillations are deployed with temporal specificity}

Next, we assessed whether the spatially specific modulation of sensorimotor oscillations is deployed with temporal specificity. For this, we restricted our analysis to those trials in which the tactile stimulus was presented after $3 \mathrm{~s}$ and thus had been expected at 1, 2, and $3 \mathrm{~s}$ (three-point hazard rate) or at 1 and $3 \mathrm{~s}$ (two-point hazard rate). Crucially, the stimulus was never expected between these three time points. To assess temporal specificity, we compared the two hazard rate conditions as well as the time points within each of the two hazard rate conditions.

Figure 3 shows the time courses of the alpha-band (Fig. $3 A$ ) and beta-band (Fig. $3 B$ ) contra-over-ipsi ratios of oscillatory power, separately for the two hazard rates. Comparing the two hazard rates by means of a cluster-based permutation test (see Materials and Methods) yielded a significant cluster $(p<0.05)$ exactly in the time window when the two hazard rates differ. However, this significant difference was only observed for beta-band oscillations (Fig. 3B). This shows that the contralateral suppression of beta-band oscillations is determined not only by a cue that directs spatial attention but also by whether or not an event is expected at a specific moment in time. Note that this difference cannot be explained by the auditory pacing tones, because these occurred identically in the two- and three-point hazard rates.

Comparing the contra-over-ipsi power ratios within each hazard rate revealed an even more striking temporal specificity. Focusing on the three-point hazard rate (red line), we observe that the beta lateralization (Fig. $3 B$ ) is strongest just before each expected event and relaxes between them. To assess this pattern quantitatively, we statistically compared the power ratios between two sets of time windows: time windows before expected events and time windows between expected events (see Materials and Methods). This comparison showed a significant effect of temporal specificity $\left(t_{(19)}=-3.12 ; p<0.01\right)$. This effect was even stronger in the two-point hazard rate condition (blue line; $\left.t_{(19)}=-5.20 ; p<0.001\right)$. Interestingly, for the alpha lateralizaand subsequently averaged.
B
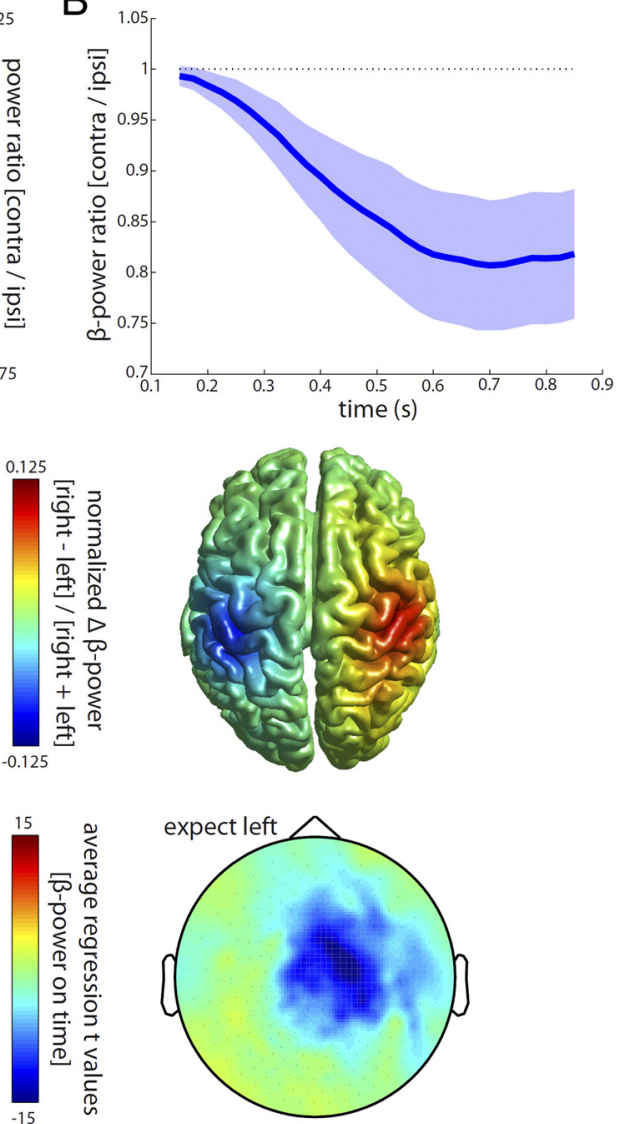

Figure 2. Spatial orienting involves a lateralization of beta-band oscillations. $\boldsymbol{A}$, Time-resolved power ratio between sensorimotor channels contralateral and ipsilateral to the anticipated tactile stimulus (see Materials and Methods). At time 0, the cue is

tion (Fig. 3A), a significant temporal modulation was observed only in the two-point hazard rate condition (blue line; $t_{(19)}=$ $-2.79 ; p<0.05)$. Thus, alpha lateralization relaxes when successive time points of interest are spaced by $2 \mathrm{~s}$ (but not when these are spaced by only $1 \mathrm{~s}$ ). These results show that spatially specific modulations of ongoing oscillations track the temporal positions at which an event is expected to occur, and that this temporal specificity is more pronounced for sensorimotor beta-band compared with alpha-band oscillations.

Anticipatory modulation of oscillatory power was also quantified by separate contra-over-baseline and ipsi-over-baseline power ratios. Outcomes of these analyses are depicted in supplemental Figure 7 (available at www.jneurosci.org as supplemental material).

Beta-suppression over contralateral sensorimotor cortex is associated with faster responses to subsequently presented tactile stimuli

We were interested in whether the observed contralateral alpha and beta suppression also leads to better task performance. For this, we separated trials with correct and incorrect responses as well as with a slow and a fast response (based on a median split). 
A

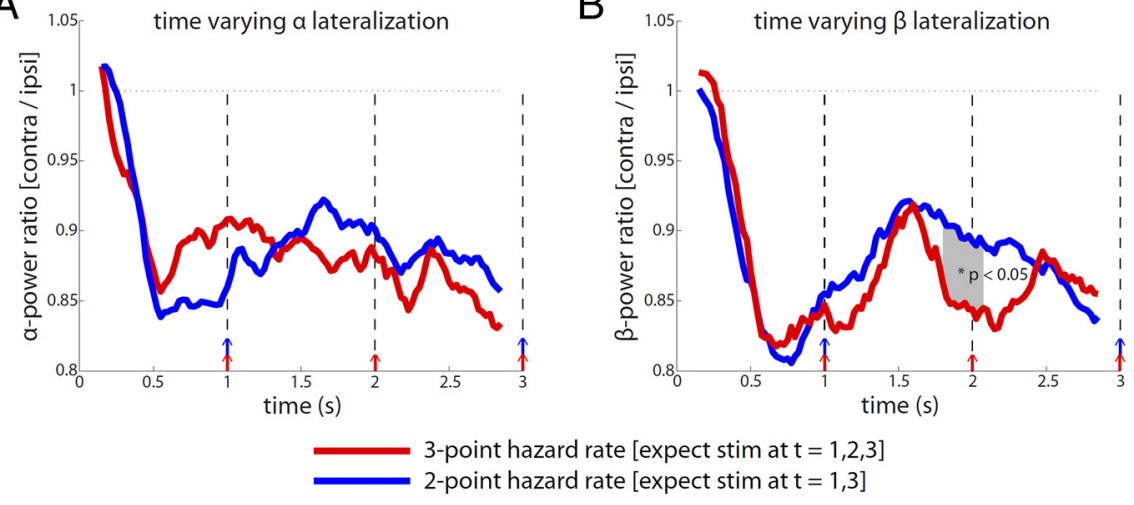

Figure 3. Temporal orienting modulates the lateralization of alpha- and beta-band oscillations. $A$, Time-resolved ratio of contralateral and ipsilateral alpha power, for both hazard rates. $\boldsymbol{B}$, Same as $\boldsymbol{A}$ but now for beta power. Shading indicates the time window for which the two hazard rates significantly differ (permutation test, $p<0.05$ ). Dashed lines indicate the three temporal positions at which tactile stimuli were expected. Blue and red arrows indicate whether a stimulus was expected in the corresponding hazard rate. Note that the depicted data are only from those trials in which the tactile stimulus occurred $3 \mathrm{~s}$ after the cue.

No significant differences were found between trials separated on the basis of accuracy. However, significant differences were found between trials separated on the basis of response time. This was the case only so for beta-band oscillations, on which we report below.

Figure $4 \mathrm{~A}$ shows the contra-over-ipsi beta power ratio for the second before the tactile event to which the response was made, separately for the fast (on average, $723 \mathrm{~ms}$ ) and the slow (on average, $1329 \mathrm{~ms}$ ) responses. Fast responses are preceded by a significantly lower contra-over-ipsi beta power ratio compared with the slow responses. The cluster-based permutation test identified two significant clusters $(p<0.01)$ of which one extends up to $1 \mathrm{~s}$ before the stimulus. Figure $4 B$ shows the topographies of the differential prestimulus beta power for the fast and slow responses, separately for subsequent left- and right-hand stimuli. Figure $4 C$ shows the corresponding source reconstructed images. These figures show that fast compared with slow responses are associated with lower prestimulus beta power over primarily the contralateral sensorimotor cortex.

This relation between response time and the contra-over-ipsi beta power ratio does not reflect motor anticipation. In fact, before the stimulus, motor preparation cannot be lateralized. Moreover, the relation between response time and the contraover-ipsi beta power ratio is not simply mediated by temporal position. In fact, although response times decreased with the position of the stimulus in the trial, the prestimulus contra-overipsi beta power ratio did not change across the possible positions of the stimulus, as can be observed in Figure $3 B$. Thus, we conclude that prestimulus contralateral beta power relates to the efficacy by which the upcoming tactile stimulus can be processed and subsequently responded to.

\section{Discussion}

We set out to investigate whether anticipation of a tactile event involves a spatially as well as temporally specific modulation of ongoing alpha- and beta-band oscillations within sensorimotor cortex. We observed three phenomena. First, orienting to an upcoming tactile event involves a spatially specific modulation of alpha- and beta-band oscillations within sensorimotor cortex. This modulation is most prominent for beta-band oscillations and is constituted by a contralateral suppression. Second, this modulation is deployed not only with spatial but also with tem- poral specificity. This temporal specificity is more pronounced for beta-band compared with alpha-band oscillations. Third, the contralateral suppression of betaband oscillations is associated with faster responses to subsequently presented tactile stimuli. These observations cannot be explained by motor preparation or execution and are thus associated with preparation for the upcoming sensory event.

Knowing when an event is likely to occur facilitates its perception, a phenomenon that is accounted for by temporal orienting of attention (Nobre, 2001). This temporally selective form of attention is associated with activation of numerous brain areas (Coull and Nobre, 1998), modulation of event-related potentials (Miniussi et al., 1999) and firing rates (Ghose and Maunsell, 2002), and a phase alignment of delta-band oscillations (Lakatos et al., 2008). We show that temporal orienting of attention also involves a temporally specific modulation of the prestimulus amplitudes of ongoing oscillations within sensory cortex. This prestimulus modulation may underlie the observed poststimulus modulations of both eventrelated potentials (Miniussi et al., 1999) and firing rates (Ghose and Maunsell, 2002), as well as the facilitation of perception that is produced by temporal orienting of attention (Nobre, 2001). Although the former pertain to an open question, we provide evidence for the latter. In fact, prestimulus suppression of betaband oscillations within contralateral sensorimotor cortex is associated with faster responses to subsequently presented tactile stimuli. We believe that these faster responses result from more efficient processing of the tactile input when these arrive during a state of suppressed beta-band oscillations. Because this state is most pronounced at times when stimuli are expected to occur, the observed modulation constitutes a mechanism by which temporal orienting of attention can facilitate perception.

Compared with temporally selective attention, spatially selective attention has been studied more extensively. Several studies have shown that spatial orienting of attention involves spatially specific modulations of ongoing oscillatory activity within sensory cortices. This phenomenon is well established for orienting to an upcoming visual event (Worden et al., 2000; Sauseng et al., 2005; Kelly et al., 2006; Thut et al., 2006), and we and others have recently observed a similar modulation for orienting to an upcoming tactile event (van Ede et al., 2010; Jones et al., 2010). The present study adds to these findings by showing that this modulation (1) is flexibly modulated by temporal predictability, (2) is constituted by a contralateral suppression, (3) occurs in both the alpha and the beta bands, and (4) improves behavioral performance in terms of response times. To complete the picture, a recent study has shown modulations of alpha-band oscillations associated with feature-specific anticipation (Snyder and Foxe, 2010). In summary, there is good empirical evidence for a general mechanism of attentional orienting that deploys anticipatory modulation of ongoing oscillations across both modalities (visual, somatosensory) and dimensions (space, time, feature).

Alpha- and beta-band modulations are characterized by a different degree of temporal specificity. In contrast, the spatially specific modulations of the alpha- and the beta-band oscillations 

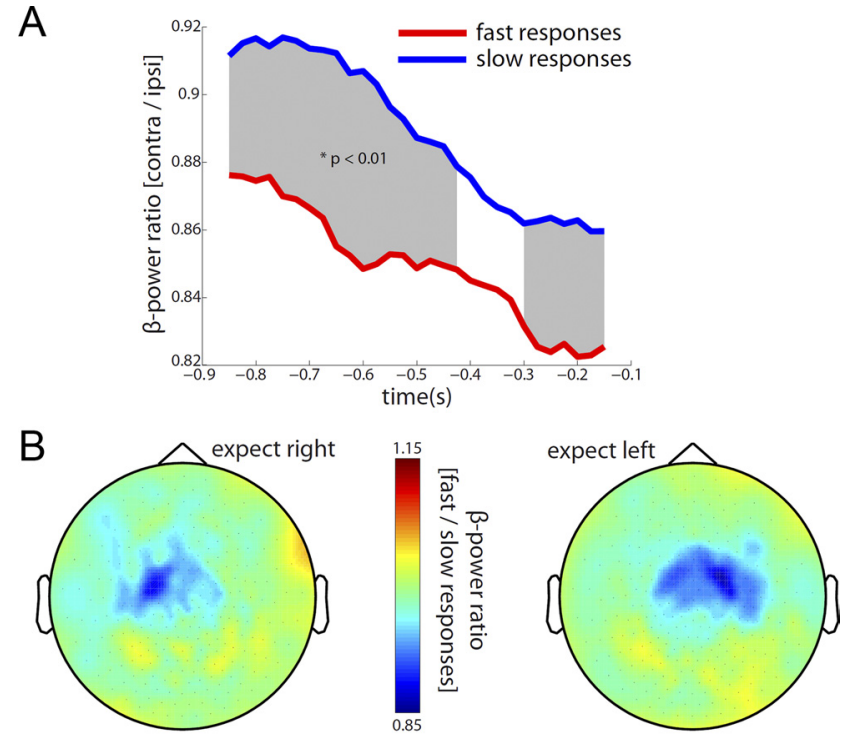

C
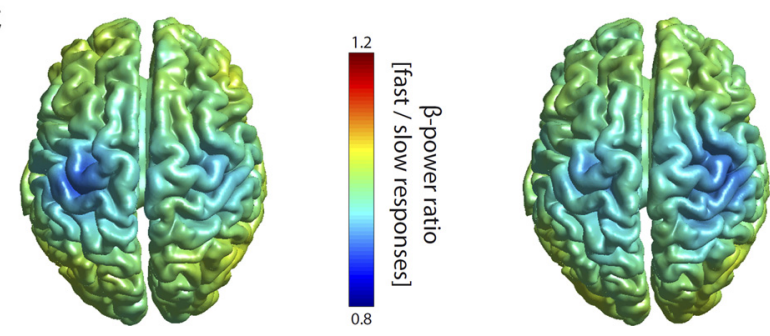

Figure 4. The contralateral prestimulus beta suppression is associated with faster responses to tactile stimuli. $\boldsymbol{A}$, Ratio between contralateral and ipsilateral beta power, separately for fast and slow responses. Fast and slow responses were separated by a participant-specific median split on the response times. Shading indicates the time windows for which slow and fast responses differ significantly (permutation test, $p<0.01$ ). $\boldsymbol{B}$, Topographies of the prestimulus beta power ratio between fast and slow responses (fast/slow), separately for anticipation of left-and right-hand tactile stimuli. Beta power was estimated for the last $350 \mathrm{~ms}$ preceding the tactile stimulus. $\boldsymbol{C}$, Identical to $\boldsymbol{B}$, except that the prestimulus beta power ratio between fast and slow responses was calculated in source space.

are highly similar. Evidence for the different degree of temporal specificity is that only for beta-band oscillations could a difference be observed between the two hazard rates. Moreover, although the lateralized modulation of beta-band oscillations relaxed between two successive anticipated events that are spaced by only $1 \mathrm{~s}$, the lateralized modulation of alpha-band oscillations relaxed only between two successive anticipated events that are spaced by $2 \mathrm{~s}$. The difference may originate from a different physiological origin of the two sensorimotor oscillations. In fact, in a modeling study, Jones et al. (2009) showed that, whereas alphaband oscillations may be produced by a thalamocortical feedforward drive, beta-band oscillations require an additional feedback drive from higher-order cortical areas. However, it cannot be ruled out that the observed difference between alpha- and betaband oscillations resulted from our data analysis being more sensitive to the beta band (see Materials and Methods). Despite this possible data-analytic disadvantage for alpha-band oscillations, we did observe a robust spatially specific modulation of alphaband oscillations. For this reason, we conclude that the observed difference in temporal specificity between the alpha and beta modulation is of physiological origin. An open question pertains to whether these differences between alpha- and beta-band modulations are restricted to tactile anticipation or are likewise observed in anticipation of sensory events in different modalities.
An additional open question pertains to whether a similar degree of temporal specificity would have been obtained if we had not used pacing tones to induce temporal expectancy.

Related to the functional differentiation of alpha- and betaband oscillations with respect to somatosensation is the issue of their localization. Traditionally, rolandic alpha is considered to be primarily a postcentral rhythm, whereas rolandic beta is considered to be primarily a precentral rhythm (Salmelin and Hari, 1994). Accordingly, beta is attributed to the motor and alpha to the somatosensory system. This notion is in conflict with our observation of beta-band oscillations as an index of somatosensory anticipation. However, recent evidence suggests that this traditional view is probably an oversimplification. First, tactile stimulation induces changes in beta power (Salenius et al., 1997; Cheyne et al., 2003; Bauer et al., 2006; Gaetz and Cheyne, 2006). Second, intracranial electrophysiological measurements directly on the postcentral gyrus have shown beta-band oscillations that originated from S1 (Crone et al., 1998; Brovelli et al., 2004). Witham and Baker (2007) even recorded stronger beta-band oscillations in S1 than in the primary motor cortex. Third, prestimulus and poststimulus beta-band oscillations are related to detectability of tactile stimuli (Linkenkaer-Hansen et al., 2004; Palva et al., 2005; Jones et al., 2010) as well as the speed with which tactile stimuli are responded to (present study). All three sets of observations show that beta-band oscillations play a role in somatosensation. Furthermore, from a more theoretical perspective, Engel and Fries (2010) propose that beta-band oscillations play a central role in the maintenance of cognitive and sensorimotor states, both within and outside of the motor system. They hypothesize that anticipation of a change is associated with a suppression of beta-band and a concomitant increase in gammaband oscillations. Although our data do not support their prediction with respect to gamma, they are fully in line with their prediction with respect to beta.

A fundamental question pertains to the mechanism by which the prestimulus suppression of ongoing alpha- and beta-band oscillations facilitates perception. Although there is ample evidence that these oscillations relate inversely to both excitability (Chen et al., 1998; Sauseng et al., 2005; Tamura et al., 2005; Romei et al., 2008) as well as the efficacy with which information is processed (Ergenoglu et al., 2004; Linkenkaer-Hansen et al., 2004; Thut et al., 2006; Hanslmayr et al., 2007; van Dijk et al., 2008; Zhang et al., 2008; Romei et al., 2010), it is at present an open question what the underlying mechanisms are. In the following, we speculate about a neurophysiological mechanism by which a prestimulus suppression of alpha- and beta-band oscillations can facilitate perception. Reductions in EEG/MEG oscillatory power are commonly interpreted as the result of a desynchronization of underlying neuronal activity (Pfurtscheller and Lopes da Silva, 1999). Accordingly, our observations might reflect the instantiation of a desynchronized neural context that allows for enhanced processing of future sensory input. This may occur via both intra-areal and inter-areal processes. With regard to the former, theoretical analysis has shown that the signal-tonoise ratio of pooled neuronal activity increases when the neuronal population desynchronizes (Zohary et al., 1994). Along these lines, attention has been shown to decorrelate neuronal activity in monkey visual cortical area V4 (Cohen and Maunsell, 2009; Mitchell et al., 2009). Between cortical areas, an efficacious neural context may be provided by selective oscillatory coupling between upstream and downstream populations (Fries, 2005). Selective neuronal gating occurs when multiple competing 
upstream populations do not oscillate in synchrony (i.e., are desynchronized), allowing only the selected (attended) population to engage in information exchange with the downstream population (Fries, 2005). We thus speculate that the suppression of alpha- and beta-band oscillatory power may reflect a desynchronization of the underlying neuronal populations that (1) increases the signal-to-noise ratio of the pooled neuronal activity (within S1) and (2) allows for a selective gating of neuronal activity (e.g., between S1 and S2). Future work is required to test and understand the physiological mechanisms underlying the anticipatory power reduction reported here.

In conclusion, anticipation of a tactile event involves a spatially as well as temporally specific modulation of ongoing sensorimotor alpha- and beta-band oscillations. The modulation of ongoing oscillations within sensory cortex may therefore constitute a unifying mechanism underlying both spatial and temporal orienting of attention.

\section{References}

Bastiaansen MC, Knösche TR (2000) Tangential derivative mapping of axial MEG applied to event-related desynchronization research. Clin Neurophysiol 111:1300-1305.

Bauer M, Oostenveld R, Peeters M, Fries P (2006) Tactile spatial attention enhances gamma-band activity in somatosensory cortex and reduces lowfrequency activity in parieto-occipital areas. J Neurosci 26:490-501.

Brovelli A, Ding M, Ledberg A, Chen Y, Nakamura R, Bressler SL (2004) beta oscillations in a large-scale sensorimotor cortical network: directional influences revealed by Granger causality. Proc Natl Acad Sci U S A 101:9849-9854.

Chen R, Yaseen Z, Cohen LG, Hallett M (1998) Time course of corticospinal excitability in reaction time and self-paced movements. Ann Neurol 44:317-325.

Cheyne D, Gaetz W, Garnero L, Lachaux JP, Ducorps A, Schwartz D, Varela FJ (2003) Neuromagnetic imaging of cortical oscillations accompanying tactile stimulation. Brain Res Cogn Brain Res 17:599-611.

Cohen MR, Maunsell JH (2009) Attention improves performance primarily by reducing interneuronal correlations. Nat Neurosci 12:1594-1600.

Coull JT, Nobre AC (1998) Where and when to pay attention: the neural systems for directing attention to spatial locations and to time intervals as revealed by both PET and fMRI. J Neurosci 18:7426-7435.

Crone NE, Miglioretti DL, Gordon B, Sieracki JM, Wilson MT, Uematsu S, Lesser RP (1998) Functional mapping of human sensorimotor cortex with electrocorticographic spectral analysis. I. alpha and beta eventrelated desynchronization. Brain 121:2271-2299.

Engel AK, Fries P (2010) beta-band oscillations: signalling the status quo? Curr Opin Neurobiol 20:156-165.

Ergenoglu T, Demiralp T, Bayraktaroglu Z, Ergen M, Beydagi H, Uresin Y (2004) alpha rhythm of the EEG modulates visual detection performance in humans. Brain Res Cogn Brain Res 20:376-383.

Fries P (2005) A mechanism for cognitive dynamics: neuronal communication through neuronal coherence. Trends Cogn Sci 9:474-480.

Gaetz W, Cheyne D (2006) Localization of sensorimotor cortical rhythms induced by tactile stimulation using spatially filtered MEG. Neuroimage 30:899-908.

Ghose GM, Maunsell JH (2002) Attentional modulation in visual cortex depends on task timing. Nature 419:616-620.

Gross J, Kujala J, Hamalainen M, Timmermann L, Schnitzler A, Salmelin R (2001) Dynamic imaging of coherent sources: studying neural interactions in the human brain. Proc Natl Acad Sci U S A 98:694-699.

Hämäläinen M, Hari R, Ilmoniemi RJ, Knuutila J, Lounasmaa OV (1993) Magnetoencephalography: theory, instrumentation, and applications to noninvasive studies of the working human brain. Rev Modern Phys 65:413-497.

Hanslmayr S, Aslan A, Staudigl T, Klimesch W, Herrmann CS, Bäuml KH (2007) Prestimulus oscillations predict visual perception performance between and within subjects. Neuroimage 37:1465-1473.
Jones SR, Pritchett DL, Sikora MA, Stufflebeam SM, Hämäläinen M, Moore CI (2009) Quantitative analysis and biophysically realistic neural modeling of the MEG mu rhythm: rhythmogenesis and modulation of sensory-evoked responses. J Neurophysiol 102:3554-3572.

Jones SR, Kerr CE, Wan Q, Pritchett DL, Hämäläinen M, Moore CI (2010) Cued spatial attention drives functionally relevant modulation of the mu rhythm in primary somatosensory cortex. J Neurosci 30:13760-13765.

Kelly SP, Lalor EC, Reilly RB, Foxe JJ (2006) Increases in alpha oscillatory power reflect an active retinotopic mechanism for distracter suppression during sustained visuospatial attention. J Neurophysiol 95:3844-3851.

Lakatos P, Karmos G, Mehta AD, Ulbert I, Schroeder CE (2008) Entrainment of neuronal oscillations as a mechanism of attentional selection. Science 320:110-113.

Liljeström M, Kujala J, Jensen O, Salmelin R (2005) Neuromagnetic localization of rhythmic activity in the human brain: a comparison of three methods. Neuroimage 25:734-745.

Linkenkaer-Hansen K, Nikulin VV, Palva S, Ilmoniemi RJ, Palva JM (2004) Prestimulus oscillations enhance psychophysical performance in humans. J Neurosci 24:10186-10190.

Maris E, Oostenveld R (2007) Nonparametric statistical testing of EEG- and MEG-data. J Neurosci Methods 164:177-190.

Miniussi C, Wilding EL, Coull JT, Nobre AC (1999) Orienting attention in time. Modulation of brain potentials. Brain 122:1507-1518

Mitchell JF, Sundberg KA, Reynolds JH (2009) Spatial attention decorrelates intrinsic activity fluctuations in macaque area V4. Neuron 63:879-888.

Nobre AC (2001) Orienting attention to instants in time. Neuropsychologia 39:1317-1328.

Nobre A, Correa A, Coull J (2007) The hazards of time. Curr Opin Neurobiol 17:465-470.

Nolte G (2003) The magnetic lead field theorem in the quasi-static approximation and its use for magnetoencephalography forward calculation in realistic volume conductors. Phys Med Biol 48:3637-3652.

Palva S, Linkenkaer-Hansen K, Näätänen R, Palva JM (2005) Early neural correlates of conscious somatosensory perception. J Neurosci 25:5248-5258.

Percival DB, Walden AT (1993) Spectral analysis for physical applications: multitaper and conventional univariate techniques. Cambridge, MA: Cambridge UP.

Pfurtscheller G, Lopes da Silva FH (1999) Event-related EEG/MEG synchronization and desynchronization: basic principles. Clin Neurophysiol 110:1842-1857.

Pogosyan A, Gaynor LD, Eusebio A, Brown P (2009) Boosting cortical activity at beta-band frequencies slows movement in humans. Curr Biol 19:1637-1641.

Romei V, Brodbeck V, Michel C, Amedi A, Pascual-Leone A, Thut G (2008) Spontaneous fluctuations in posterior alpha-band EEG activity reflect variability in excitability of human visual areas. Cereb Cortex 18:2010-2018.

Romei V, Gross J, Thut G (2010) On the role of prestimulus alpha rhythms over occipito-parietal areas in visual input regulation: correlation or causation? J Neurosci 30:8692-8697.

Salenius S, Schnitzler A, Salmelin R, Jousmäki V, Hari R (1997) Modulation of human cortical rolandic rhythms during natural sensorimotor tasks. Neuroimage 5:221-228.

Salmelin R, Hari R (1994) Spatiotemporal characteristics of sensorimotor neuromagnetic rhythms related to thumb movement. Neuroscience 60:537-550.

Sauseng P, Klimesch W, Stadler W, Schabus M, Doppelmayr M, Hanslmayr S, Gruber WR, Birbaumer N (2005) A shift of visual spatial attention is selectively associated with human EEG alpha activity. Eur J Neurosci 22:2917-2926.

Sauseng P, Klimesch W, Gerloff C, Hummel FC (2009) Spontaneous locally restricted EEG alpha activity determines cortical excitability in the motor cortex. Neuropsychologia 47:284-288.

Snyder AC, Foxe JJ (2010) Anticipatory attentional suppression of visual features indexed by oscillatory alpha-band power increases: a highdensity electrical mapping study. J Neurosci 30:4024-4032.

Tamura Y, Hoshiyama M, Nakata H, Hiroe N, Inui K, Kaneoke Y, Inoue K, Kakigi R (2005) Functional relationship between human rolandic 
oscillations and motor cortical excitability: an MEG study. Eur J Neurosci 21:2555-2562.

Thut G, Nietzel A, Brandt SA, Pascual-Leone A (2006) alpha-band electroencephalographic activity over occipital cortex indexes visuospatial attention bias and predicts visual target detection. J Neurosci 26:9494-9502.

van Dijk H, Schoffelen JM, Oostenveld R, Jensen O (2008) Prestimulus oscillatory activity in the alpha band predicts visual discrimination ability. J Neurosci 28:1816-1823.

van Ede F, Jensen O, Maris E (2010) Tactile expectation modulates prestimulus beta-band oscillations in human sensorimotor cortex. Neuroimage 51:867-876.
Witham CL, Baker SN (2007) Network oscillations and intrinsic spiking rhythmicity do not covary in monkey sensorimotor areas. J Physiol 580:801-814

Worden MS, Foxe JJ, Wang N, Simpson GV (2000) Anticipatory biasing of visuospatial attention indexed by retinotopically specific alpha-band electroencephalography increases over occipital cortex. J Neurosci 20:RC63(1-6).

Zhang Y, Wang X, Bressler SL, Chen Y, Ding M (2008) Prestimulus cortical activity is correlated with speed of visuomotor processing. J Cogn Neurosci 20:1915-1925.

Zohary E, Shadlen MN, Newsome WT (1994) Correlated neuronal discharge rate and its implications for psychophysical performance. Nature 370:140-143. 